\title{
Projection onto simplicial cones by a semi-smooth Newton method *
}

\author{
O. P. Ferreira ${ }^{\dagger} \quad$ S. Z. Németh ${ }^{\ddagger}$
}

August 27, 2018

\begin{abstract}
By using Moreau's decomposition theorem for projecting onto cones, the problem of projecting onto a simplicial cone is reduced to finding the unique solution of a nonsmooth system of equations. It is shown that a semi-smooth Newton method applied to the system of equations associated to the problem of projecting onto a simplicial cone is always well defined, and the generated sequence is bounded for any starting point and under a somewhat restrictive assumption it is finite. Besides, under a mild assumption on the simplicial cone, the generated sequence converges linearly to the solution of the associated system of equations.
\end{abstract}

\section{Introduction}

The interest in the subject of projection arises in several situations, having a wide range of applications in pure and applied mathematics such as Convex Analysis (see e.g. [12]), Optimization (see e.g. [2], 4], [5], [11, [24], [26]), Numerical Linear Algebra (see e.g. [25]), Statistics (see e.g. 3], 8], [14]), Computer Graphics (see e.g. [10] ) and Ordered Vector Spaces (see e.g. [1, [16], [17], 21], 22], [23]). More specifically, the projection onto a polyhedral cone, which has as a special case the projection onto a simplicial one, is a problem of high impact on scientific community 1 . The geometric nature of this problem makes it particularly interesting and important in many areas of science and technology such as Statistics (see e.g. [14]), Computation (see e.g. [15]), Optimization (see e.g. [20], [26]) and Ordered Vector Spaces (see e.g. 21]).

In this paper we particularize the Moreau's decomposition theorem for simplicial cones. This leads to an equivalence between the problem of projecting a point onto a simplicial cone and the one of finding the unique solution of a nonsmooth system of equations. We apply a semi-smooth Newton method for finding a unique solution of the obtained associated system. We show that

*1991 A M S Subject Classification. Primary 90C33; Secondary 15A48, Key words and phrases. Metric projection onto simplicial cones

${ }^{\dagger}$ IME/UFG, Campus II- Caixa Postal 131, Goiânia, GO, 74001-970, Brazil (e-mail:orizon@ufg.br). The author was supported in part by FAPEG, CNPq Grants 471815/2012-8, 303732/2011-3 and PRONEXOptimization(FAPERJ/CNPq).

${ }^{\ddagger}$ School of Mathematics, The University of Birmingham, The Watson Building, Edgbaston, Birmingham B15 2TT, United Kingdom (e-mail:nemeths@for.mat.bham.ac.uk). The author was supported in part by the Hungarian Research Grant OTKA 60480.

${ }^{1}$ see the popularity of the Wikimization page Projection on Polyhedral Cone at
http://www.convexoptimization.com/wikimization/index.php/Special:Popularpages


the method is always well defined and the generated sequence is bounded for any starting point, and under a somewhat restrictive assumption it is finite. Besides, under a mild assumption on the simplicial cone, the generated sequence converges linearly to the solution of the associated system of equations. It is worth pointing out that a similar approach has been considered by Mangasarian in [18] for finding solutions of NP-hard absolute value equations.

\section{Preliminaries}

Consider $\mathbb{R}^{m}$ endowed with an orthogonal coordinate system and let $\langle\cdot, \cdot\rangle$ be the canonical scalar product defined by it. Denote by $\|\cdot\|$ be the norm generated by $\langle\cdot, \cdot\rangle$. If $a \in \mathbb{R}$ and $x=\left(x^{1}, \ldots, x^{m}\right) \in$ $\mathbb{R}^{m}$, then denote $a^{+}:=\max \{a, 0\}, a^{-}:=\max \{-a, 0\}$ and

$$
x^{+}:=\left(\left(x^{1}\right)^{+}, \ldots,\left(x^{m}\right)^{+}\right) .
$$

For $x \in \mathbb{R}^{m}$, the vector $\operatorname{sgn}(x)$ will denote a vector with components equal to 1,0 or -1 depending on whether the corresponding component of the vector $x$ is positive, zero or negative. We will call a closed set $K \subset \mathbb{R}^{m}$ a cone if the following conditions hold:

(i) $\lambda x+\mu y \in K$ for any $\lambda, \mu \geq 0$ and $x, y \in K$,

(ii) $x,-x \in K$ implies $x=0$.

Let $K \subset \mathbb{R}^{n}$ be a closed convex cone. The polar cone of $K$ is the set

$$
K^{\perp}:=\left\{x \in \mathbb{R}^{n} \mid\langle x, y\rangle \leq 0, \forall y \in K\right\} .
$$

For any positive integer $p$ denote by $I_{p}$ the $p \times p$ identity matrix. Denote $I_{m}=I$ and $\operatorname{diag}(x)$ will denote a diagonal matrix corresponding to elements of $x$. For an $m \times m$ matrix $M$ consider the norm defined by $\|M\|:=\max _{x \neq 0}\{\|M x\|:\|x\|=1\}$, this definition implies

$$
\|M x\| \leq\|M\|\|x\|, \quad\|L M\| \leq\|L\|\|M\|,
$$

for any $m \times m$ matrices $L$ and $M$.

The following lemma is Theorem 3.1.4 on page 45 of [7].

Lemma 1 (Banach's Lemma) Let $E$ be $m \times m$ matrix and $I$ the $m \times m$ identity matrix. If $\|E\|<1$, then $E-I$ is invertible and $\left\|(E-I)^{-1}\right\| \leq 1 /(1-\|E\|)$.

Denote $\mathbb{R}_{+}^{m}=\left\{x=\left(x^{1}, \ldots, x^{m}\right) \in \mathbb{R}^{m}: x_{1} \geq 0, \ldots, x^{m} \geq 0\right\}$ the nonnegative orthant. Let $A$ be an $m \times m$ nonsingular matrix. Then, the cone

$$
K:=A \mathbb{R}_{+}^{m},
$$

is called a simplicial cone. Let $z \in \mathbb{R}^{m}$, then the projection $P_{K}(z)$ of the point $z$ onto the cone $K$ is defined by

$$
P_{K}(z):=\operatorname{argmin}\{\|z-y\|: y \in K\} .
$$


Remark 1 It easy to see that $P_{\mathbb{R}_{+}^{m}}(z)=z^{+}$and it is well know that the projection onto a convex set is continuous and nonexpansive, see [12].

The above remark shows that projection onto the nonnegative orthant is an easy problem. On the other hand, the projection onto a general simplicial cone is difficult and computationally expensive, this problem has been studied e.g. in $99,11,21,26]$. The statement of the problem that we are interested is:

Problem 1 (projection onto a simplicial cone) Given $A$ an $m \times m$ nonsingular matrix and $z \in \mathbb{R}^{m}$, find the projection $P_{K}(z)$ of the point $z$ onto the simplicial cone $K=A \mathbb{R}_{+}^{m}$.

As we will see in the next section, by using Moreau's decomposition theorem for projecting onto cones, solving Problem 1 is reduced to solving the following problem.

Problem 2 (nonsmooth equation) Given $A$ an $m \times m$ nonsingular matrix and $z \in \mathbb{R}^{m}$, find the unique solution $u$ of the nonsmooth equation

$$
\left(A^{\top} A-I\right) x^{+}+x=A^{\top} z .
$$

In this case, $P_{K}(z)=A u^{+}$where $K=A \mathbb{R}_{+}^{m}$.

We will show in Section 4 that Problem 2 can be solved by using a semi-smooth Newton method.

\section{Moreau's decomposition theorem for simplicial cones}

We recall the following result of Moreau [19]:

Theorem 1 (Moreau's decomposition theorem) Let $K, L \subseteq \mathbb{R}^{m}$ be two mutually polar cones in $\mathbb{R}^{m}$. Then, the following statements are equivalent:

(i) $z=x+y, x \in K, y \in L$ and $\langle x, y\rangle=0$,

(ii) $x=P_{K}(z)$ and $y=P_{L}(z)$.

The following result follows from the definition of the polar. For a proof see for example [1].

Lemma 2 Let $A$ be an $m \times m$ nonsingular matrix. Then,

$$
\left(A \mathbb{R}_{+}^{m}\right)^{\perp}=-\left(A^{\top}\right)^{-1} \mathbb{R}_{+}^{m} .
$$

The following result has been proved in [1] by using Moreau's decomposition theorem and Lemma 2 ,

Lemma 3 Let $A$ be an $m \times m$ nonsingular matrix and $K=A \mathbb{R}_{+}^{m}$ the corresponding simplicial cone. Then, for any $z \in \mathbb{R}^{m}$ there exists a unique $x \in \mathbb{R}^{m}$ such that the following two equivalent statements hold:

(i) $z=A x^{+}-\left(A^{\top}\right)^{-1} x^{-}, x \in \mathbb{R}^{m}$, 
(ii) $A x^{+}=P_{K}(z)$ and $-\left(A^{\top}\right)^{-1} x^{-}=P_{K^{\perp}}(z)$.

The following result is a direct consequence of Lemma 3, it shows that solving Problem 1 is reduced to solving Problem 2 ,

Lemma 4 Let $A$ be a nonsingular matrix, $K=A \mathbb{R}_{+}^{m}$ the corresponding simplicial cone and $z \in \mathbb{R}^{m}$ arbitrary. Then, equation (2) has a unique solution $u$ and $P_{K}(z)=A u^{+}$.

Proof. Since $A$ is an $m \times m$ nonsingular matrix, multiplying by $A^{\top}$, item (i) of Lemma 3 is equivalently transformed into

$$
A^{\top} A x^{+}-x^{-}=A^{\top} z .
$$

As $-x^{-}=x-x^{+}$, the above equation is equivalent to (2). Therefore, equation (2) is equivalent to the equation in item (i) of Lemma 3. Hence, we conclude from Lemma 3 that equation (2) has a unique solution $u$ and $P_{K}(z)=A u^{+}$.

\section{Semi-smooth Newton method}

The semi-smooth Newton iteration for solving equation (2) or equivalently for finding the zero of the function

$$
F(x):=\left(A^{\top} A-I\right) x^{+}+x-A^{\top} z,
$$

is formally defined by

$$
F\left(x_{k}\right)+S_{k}\left(x_{k+1}-x_{k}\right)=0, \quad S_{k} \in \partial F\left(x_{k}\right), \quad k=0,1,2, \ldots,
$$

where $\partial F\left(x_{k}\right)$ denotes the Clarke generalized Jacobian of $F$ at $x_{k}$. From the definition of Clarke generalized Jacobian of the function $F$ at $x$ (see [6]) it easy to conclude that

$$
\left(A^{\top} A-I\right) \operatorname{diag}\left(\operatorname{sgn}\left(x^{+}\right)\right)+I \in \partial F(x) .
$$

Therefore, by using the last inclusion, the semi-smooth Newton iteration (3) reduces to

$$
\left(A^{\top} A-I\right) x_{k}^{+}+x_{k}-A^{\top} z+\left(\left(A^{\top} A-I\right) \operatorname{diag}\left(\operatorname{sgn}\left(x_{k}^{+}\right)\right)+I\right)\left(x_{k+1}-x_{k}\right)=0,
$$

and as $\operatorname{diag}\left(\operatorname{sgn}\left(x_{k}^{+}\right)\right) x_{k}=x_{k}^{+}$, the latter equality becomes

$$
\left(\left(A^{\top} A-I\right) \operatorname{diag}\left(\operatorname{sgn}\left(x_{k}^{+}\right)\right)+I\right) x_{k+1}=A^{\top} z, \quad k=0,1,2, \ldots,
$$

which formally defines a sequence $\left\{x_{k}\right\}$ with starting point $x_{0}$, called the semi-smooth Newton sequence for solving equation (2) or for projecting a point $z \in \mathbb{R}^{m}$ onto the simplicial cone $K$.

Lemma 5 Let $A$ be an $m \times m$ nonsingular matrix. Then the matrix

$$
\left(A^{\top} A-I\right) \operatorname{diag}\left(\operatorname{sgn}\left(x^{+}\right)\right)+I,
$$

is nonsingular for all $x \in \mathbb{R}^{m}$. As a consequence, the semi-smooth Newton sequence $\left\{x_{k}\right\}$ is well defined from any starting point. 
Proof. To simplify the notations define $B=A^{\top} A$. Thus, the matrix in (5) becomes

$$
C:=(B-I) \operatorname{diag}\left(\operatorname{sgn}\left(x^{+}\right)\right)+I .
$$

Hence, to prove the first part of the lemma we need to show that $C$ is a nonsingular matrix. Let $x=\left(x^{1}, \ldots, x^{m}\right)$ and the sets $P(x)=\left\{i \in\{1, \ldots, m\}: x^{i}>0\right\}$ and $\tilde{P}(x)=\{1, \ldots, m\} \backslash P(x)$. For an $m \times m$ matrix $M$ define $M^{P(x)}$ and $M^{\tilde{P}(x)}$ the matrices defined, respectively, by

$$
M_{i j}^{P(x)}=\left\{\begin{array}{ll}
M_{i j} & \text { if } j \in P(x), \\
0 & \text { if } j \notin P(x) .
\end{array} M_{i j}^{\tilde{P}(x)}= \begin{cases}M_{i j} & \text { if } j \in \tilde{P}(x), \\
0 & \text { if } j \notin \tilde{P}(x) .\end{cases}\right.
$$

Let $k:=m-|P(x)|$. Then, from the definitions of the matrix $C$ and the index sets $P(x)$ and $\tilde{P}(x)$, it is easy to see that

$$
C=B^{P(x)}+I^{\tilde{P}(x)}
$$

and there is an $m \times m$ permutation matrix $\Pi$ such that $\Pi^{\top} C \Pi$ is upper block triangular of the form

$$
\Pi^{\top} C \Pi=\left[\begin{array}{cc}
I_{k} & E \\
0 & F
\end{array}\right]
$$

where $F$ is a principal submatrix of $B$. Since $A$ is nonsingular, $B=A^{\top} A$ is positive definite and $F$ is a principal submatrix of $B$, we conclude that the principal minor $\operatorname{det} F \neq 0$ (see Corollary 7.1.5 of [13]). Hence, using (6) it follows that

$$
\operatorname{det}\left(\Pi^{\top}\right) \operatorname{det} C \operatorname{det} \Pi=\operatorname{det}\left(\Pi^{\top} C \Pi\right)=\operatorname{det} I_{k} \operatorname{det} F=\operatorname{det} F \neq 0 .
$$

As $\operatorname{det}\left(\Pi^{\top}\right)=(\operatorname{det} \Pi)^{-1}$, the last relation implies $\operatorname{det} C=\operatorname{det} F \neq 0$, hence the first part of the lemma is proven.

The proof of the second part of the lemma is immediate consequence of the definition of the sequence $\left\{x_{k}\right\}$ in (4) and the first part of the lemma.

The next proposition give a condition for the Newton iteration (44) to finish in a finite number of steps.

Proposition 1 If in (4) it happens that $\operatorname{sgn}\left(x_{k+1}^{+}\right)=\operatorname{sgn}\left(x_{k}^{+}\right)$, then $x_{k+1}$ solves equation (2) and $P_{K}(z)=A x_{k+1}^{+}$.

Proof. If $\operatorname{sgn}\left(x_{k+1}^{+}\right)=\operatorname{sgn}\left(x_{k}^{+}\right)$in equation (4), then it becomes

$$
\left(\left(A^{\top} A-I\right) \operatorname{diag}\left(\operatorname{sgn}\left(x_{k+1}^{+}\right)\right)+I\right) x_{k+1}=A^{\top} z .
$$

Since $\operatorname{diag}\left(\operatorname{sgn}\left(x_{k+1}^{+}\right)\right) x_{k+1}=x_{k+1}^{+}$, (7) yields

$$
\left(A^{\top} A-I\right) x_{k+1}^{+}+x_{k+1}=A^{\top} z,
$$

which implies that $x_{k+1}$ is a solution of (2) and, by using Lemma 4 , we have $P_{K}(z)=A x_{k+1}^{+}$.

The next proposition shows that the semi-smooth Newton sequence $\left\{x_{k}\right\}$ is bounded and gives a formula for any accumulation point of it. 
Proposition 2 The semi-smooth Newton sequence $\left\{x_{k}\right\}$ is bounded from any starting point. Moreover, for each accumulation point $\bar{x}$ of $\left\{x_{k}\right\}$ there exists $\hat{x} \in \mathbb{R}^{m}$ such that

$$
\left(\left(A^{\top} A-I\right) \operatorname{diag}\left(\operatorname{sgn}\left(\hat{x}^{+}\right)\right)+I\right) \bar{x}=A^{\top} z .
$$

Proof. First suppose that $\left\{x_{k}\right\}$ is unbounded. Then, since $\left\{x_{k}\right\}$ is unbounded, the unit sphere is compact, and there are only finitely many vectors $\operatorname{sgn}\left(x_{k}^{+}\right)$with coordinates 0 or 1 , it follows that there exists a vector $\tilde{x} \in \mathbb{R}^{m}$ and a subsequence $\left\{x_{k_{j}}\right\}$ of $\left\{x_{k}\right\}$ such that

$$
\lim _{j \rightarrow \infty}\left\|x_{k_{j}+1}\right\|=\infty, \quad \lim _{j \rightarrow \infty} \frac{x_{k_{j}+1}}{\left\|x_{k_{j}+1}\right\|}=v \neq 0, \quad \operatorname{sgn}\left(x_{k_{j}}^{+}\right) \equiv \operatorname{sgn}\left(\tilde{x}^{+}\right) .
$$

Therefore, $\operatorname{as} \operatorname{sgn}\left(x_{k_{j}}^{+}\right)=\operatorname{sgn}\left(\tilde{x}^{+}\right)$for all $j$, the definition of the semi-smooth Newton sequence $\left\{x_{k}\right\}$ in (44) implies

$$
\left(\left(A^{\top} A-I\right) \operatorname{diag}\left(\operatorname{sgn}\left(\tilde{x}^{+}\right)\right)+I\right) \frac{x_{k_{j}+1}}{\left\|x_{k_{j}+1}\right\|}=\frac{A^{\top} z}{\left\|x_{k_{j}+1}\right\|}, \quad j=0,1,2, \ldots
$$

By tending with $j$ to infinity in the above equality and by taking into account (즈), it follows that

$$
\left(\left(A^{\top} A-I\right) \operatorname{diag}\left(\operatorname{sgn}\left(\tilde{x}^{+}\right)\right)+I\right) v=0,
$$

which contradicts the first part of the Lemma 5 since $v \neq 0$. Therefore, the sequence $\left\{x_{k}\right\}$ is bounded, which proves the first part of the proposition.

For proving the second part of the lemma, let $\bar{x}$ be an accumulation point of the sequence $\left\{x_{k}\right\}$. Then, since there are only finitely many vectors $\operatorname{sgn}\left(x_{k}^{+}\right)$with coordinates 0 or 1 , there exists a vector $\hat{x} \in \mathbb{R}^{m}$ and a subsequence $\left\{x_{k_{j}}\right\}$ of $\left\{x_{k}\right\}$ such that

$$
\lim _{j \rightarrow \infty} x_{k_{j}+1}=\bar{x}, \quad \operatorname{sgn}\left(x_{k_{j}}^{+}\right) \equiv \operatorname{sgn}\left(\hat{x}^{+}\right),
$$

Since $\operatorname{sgn}\left(x_{k_{j}}^{+}\right)=\operatorname{sgn}\left(\hat{x}^{+}\right)$for all $j$, the definition of the semi-smooth Newton sequence $\left\{x_{k}\right\}$ in (4) implies

$$
\left(\left(A^{\top} A-I\right) \operatorname{diag}\left(\operatorname{sgn}\left(\hat{x}^{+}\right)\right)+I\right) x_{k_{j}+1}=A^{\top} z, \quad j=0,1,2, \ldots
$$

Taking the limit in the last equality as $k_{j}$ goes to $\infty$, the second part of the proposition follows.

The next lemma gives a condition for the convergence of the semi-smooth Newton sequence $\left\{x_{k}\right\}$.

Lemma 6 Let $a \in(0,1 / 2)$. If for any diagonal matrix $G$, with diagonal elements 0 or 1

$$
\left\|\left(\left(A^{\top} A-I\right) G+I\right)^{-1}\left(A^{\top} A-I\right)\right\|<a,
$$

then the semi-smooth Newton sequence $\left\{x_{k}\right\}$ converges linearly to the unique solution $u$ of the equation (2) from any starting point and $P_{K}(z)=A u^{+}$. 
Proof. Let $u$ be the solution of equation (2) and $\left\{x_{k}\right\}$ be the sequence defined in (4). To simplify the notations, denote

$$
B=A^{\top} A, \quad D^{k}=\operatorname{diag}\left(\operatorname{sgn}\left(x_{k}^{+}\right)\right) .
$$

Since $u$ is the solution of equation (2), using the above notations and the definition of $\left\{x_{k}\right\}$ in (4), we have

$$
(B-I) u^{+}+u=A^{\top} z, \quad\left((B-I) D^{k}+I\right) x_{k+1}=A^{\top} z .
$$

By subtracting the two equalities above, and by taking into account that $D^{k} x_{k}=x_{k}^{+}$, we obtain, after some straightforward manipulations, that

$$
(B-I)\left(x_{k}^{+}-u^{+}\right)+(B-I) D^{k}\left(x_{k+1}-x_{k}\right)+x_{k+1}-u=0,
$$

or equivalently,

$$
\left((B-I) D^{k}+I\right)\left(x_{k+1}-u\right)+(B-I)\left(x_{k}^{+}-u^{+}+D^{k}\left(u-x_{k}\right)\right)=0,
$$

Since $D^{k}=\operatorname{diag}\left(\operatorname{sgn}\left(x_{k}^{+}\right)\right)$, by using the last equality and Lemma 5 , we get

$$
x_{k+1}-u=-\left((B-I) D^{k}+I\right)^{-1}(B-I)\left(D^{k}\left(x_{k}-u\right)+\left(x_{k}^{+}-u^{+}\right)\right) .
$$

By using the first inequality in (11), the Cauchy inequality, $\left\|D^{k}\right\| \leq 1$, and the nonexpansivity of the projection mapping $x \mapsto x^{+}$onto the nonnegative orthant (see Remark 1), the latter relation and assumption (91) gives

$$
\left\|x_{k+1}-u\right\| \leq\left\|\left((B-I) D^{k}+I\right)^{-1}(B-I)\right\| 2\left\|x_{k}-u\right\|<2 a\left\|x_{k}-u\right\|,
$$

where $2 a<1$. As the last inequality holds for all $k$, we conclude that the sequence $\left\{x_{k}\right\}$ converges linearly to $u$ and Lemma 4 implies $P_{K}(z)=A u^{+}$.

The next theorem provides a sufficient condition for the linear convergence of the Newton iteration.

Theorem 2 Let $b \in(0,1 / 3)$. If

$$
\left\|A^{\top} A-I\right\|<b,
$$

then the semi-smooth Newton sequence $\left\{x_{k}\right\}$ converges linearly to the unique solution $u$ of equation (2) from any starting point and $P_{K}(z)=A u^{+}$.

Proof. To simplify the notations, denote $B=A^{\top} A$. By Lemma 6 it is enough to show that

$$
\left\|((B-I) G+I)^{-1}(B-I)\right\|<\frac{b}{1-b}<\frac{1}{2},
$$

for any diagonal matrix $G$ with diagonal elements 0 or 1 . By using the properties (11) of the norm, it is enough to show that

$$
\left\|(I-(I-B) G)^{-1}\right\|\|(I-B)\|<\frac{b}{1-b} .
$$


Since $\|G\| \leq 1$, assumption (10) and the properties (10) of the norm imply

$$
\|(I-B) G\| \leq\|I-B\|\|G\| \leq\|I-B\|<b<1 .
$$

Thus, by applying Lemma 1 with $E=(I-B) G$ and by using the last inequality, we conclude that

$$
\left\|(I-(I-B) G)^{-1}\right\|\|I-B\| \leq \frac{\|I-B\|}{1-\|(I-B) G\|}<\frac{b}{1-b},
$$

which is exactly the required relation (11). By using Lemma 4, we have $P_{K}(z)=A u^{+}$.

\section{Conclusions}

In this paper we studied the problem of projection onto a simplicial cone which, via Moreau's decomposition theorem for projecting onto cones, is reduced to finding the unique solution of a nonsmooth system of equations. Our main result shows that, under a mild assumption on the simplicial cone, we can apply a semi-smooth Newton method for finding a unique solution of the obtained associated system and that the generated sequence converges linearly to the solution for any starting point. It would be interesting to see whether the used technique can be applied for finding the projection onto more general cones. As has been shown in [26], the problem of projection onto a simplicial cone is reduced to a certain type of linear complementarity problem (LCP). Then, another interesting problem to address is to compare the four methods for projecting onto a simplicial cone, namely, semi-smooth Newton method, the methods proposed in [9, 20, 26] and the Lemke's method for LCPs.

\section{References}

[1] M. Abbas and S. Z. Németh. Solving nonlinear complementarity problems by isotonicity of the metric projection. J. Math. Anal. Appl., 386(2):882-893, 2012.

[2] H. H. Bauschke and J. M. Borwein. On projection algorithms for solving convex feasibility problems. SIAM Rev., 38(3):367-426, 1996.

[3] R. Berk and R. Marcus. Dual cones, dual norms, and simultaneous inference for partially ordered means. J. Amer. Statist. Assoc., 91(433):318-328, 1996.

[4] Y. Censor, T. Elfving, G. T. Herman, and T. Nikazad. On diagonally relaxed orthogonal projection methods. SIAM J. Sci. Comput., 30(1):473-504, 2007/08.

[5] Y. Censor, D. Gordon, and R. Gordon. Component averaging: an efficient iterative parallel algorithm for large and sparse unstructured problems. Parallel Comput., 27(6):777-808, 2001.

[6] F. H. Clarke. Optimization and nonsmooth analysis, volume 5 of Classics in Applied Mathematics. Society for Industrial and Applied Mathematics (SIAM), Philadelphia, PA, second edition, 1990. 
[7] J. E. Dennis, Jr. and R. B. Schnabel. Numerical methods for unconstrained optimization and nonlinear equations, volume 16 of Classics in Applied Mathematics. Society for Industrial and Applied Mathematics (SIAM), Philadelphia, PA, 1996. Corrected reprint of the 1983 original.

[8] R. L. Dykstra. An algorithm for restricted least squares regression. J. Amer. Statist. Assoc., 78(384):837-842, 1983.

[9] A. Ekárt, A. B. Németh, and S. Z. Németh. Rapid heuristic projection on simplicial cones, 2010, arXiv:1001.1928.

[10] J. D. Foley, A. van Dam, S. K. Feiner, and J. F. Hughes. Computer Graphics: Principles and Practice. Addison-Wesley systems programming series, 1990.

[11] H. Frick. Computing projections into cones generated by a matrix. Biometrical J., 39(8):975987, 1997.

[12] J.-B. Hiriart-Urruty and C. Lemaréchal. Convex analysis and minimization algorithms: Fundamentals. I, volume 305 of Grundlehren der Mathematischen Wissenschaften [Fundamental Principles of Mathematical Sciences]. Springer-Verlag, Berlin, 1993.

[13] R. A. Horn and C. R. Johnson. Matrix analysis. Cambridge University Press, Cambridge, 1985.

[14] X. Hu. An exact algorithm for projection onto a polyhedral cone. Aust. N. Z. J. Stat., 40(2):165-170, 1998.

[15] T. Huynh, C. Lassez, and J.-L. Lassez. Practical issues on the projection of polyhedral sets. Ann. Math. Artificial Intelligence, 6(4):295-315, 1992. Artificial intelligence and mathematics, II.

[16] G. Isac and A. B. Németh. Monotonicity of metric projections onto positive cones of ordered Euclidean spaces. Arch. Math. (Basel), 46(6):568-576, 1986.

[17] G. Isac and A. B. Németh. Isotone projection cones in Euclidean spaces. Ann. Sci. Math. Québec, 16(1):35-52, 1992.

[18] O. L. Mangasarian. A generalized Newton method for absolute value equations. Optim. Lett., 3(1):101-108, 2009.

[19] J. J. Moreau. Décomposition orthogonale d'un espace hilbertien selon deux cônes mutuellement polaires. C. R. Acad. Sci., 255:238-240, 1962.

[20] P. M. Morillas. Dykstra's algorithm with strategies for projecting onto certain polyhedral cones. Appl. Math. Comput., 167(1):635-649, 2005.

[21] A. B. Németh and S. Z. Németh. How to project onto an isotone projection cone. Linear Algebra Appl., 433(1):41-51, 2010.

[22] S. Z. Németh. Characterization of latticial cones in Hilbert spaces by isotonicity and generalized infimum. Acta Math. Hungar., 127(4):376-390, 2010. 
[23] S. Z. Németh. Isotone retraction cones in Hilbert spaces. Nonlinear Anal., 73(2):495-499, 2010 .

[24] H. D. Scolnik, N. Echebest, M. T. Guardarucci, and M. C. Vacchino. Incomplete oblique projections for solving large inconsistent linear systems. Math. Program., 111(1-2, Ser. B):273$300,2008$.

[25] G. W. Stewart. On the perturbation of pseudo-inverses, projections and linear least squares problems. SIAM Rev., 19(4):634-662, 1977.

[26] M. Ujvári. On the projection onto a finitely generated cone, 2007, Preprint WP 2007-5, MTA SZTAKI, Laboratory of Operations Research and Decision Systems, Budapest. 